\title{
Is Endometriosis a Surgical Disease?
}

\section{Paul J Yong*, Christina William and Catherine Allaire}

Department of Obstetrics \& Gynaecology, University of British Columbia; BC Women's Centre for Pelvic Pain and Endometriosis

Endometriosis affects one in ten reproductive-aged women, is a cause of pelvic pain and infertility, and accounts for $\$ 22$ billion and $\$ 1.8$ billion in total annual costs to American and Canadian society, respectively [1-3]. Endometriosis is defined as uterine endometrial glands/stroma being ectopically present outside of the uterus, usually elsewhere in the pelvis (e.g. the ovaries or pelvic peritoneum). The formal diagnosis of endometriosis requires surgical excision or biopsy followed by histological confirmation. However it is controversial whether endometriosis is truly a surgical disease.

One argument against endometriosis as a surgical disease is that formal diagnosis requires surgery and thus invasive and not without risk, and therefore national guidelines support initial empiric medical treatment of symptomatic women [1]. Nonetheless, such symptomatic women are likely to have a spectrum of underlying conditions such as endometriosis, primary dysmenorrhea, or even non-gynecologic problems. If empiric medical treatment (e.g. hormonal suppression) is unsuccessful, or if the patient chooses to discontinue medical treatment (e.g. due to side-effects or desire for pregnancy), then conservative surgery can be offered to diagnose and treat endometriosis, if present. Conservative surgery for endometriosis involves laparoscopic ablation or excision of the endometriosis lesions. In addition to its therapeutic aspect, laparoscopic surgery provides women additional information about their reproductive health. For example, if endometriosis is present then women can be counseled about implications for fertility [4], new research showing a relationship between endometriosis and ovarian cancer [5,6], and the increased risk of endometriosis for family members [7]. Correspondingly, women diagnosed with endometriosis may alter the timing of their reproductive decision making, choose long-term hormonal contraception for prevention of ovarian cancer, and their young family members may seek treatment earlier and thus possibly prevent development of chronic pelvic pain. Alternatively, if no endometriosis is found, then women can be reassured. This difference in prognosis highlights the importance of laparoscopic recognition of not only classic lesions of endometriosis (e.g. powder-burn), but also its atypical and subtle appearances (e.g. vascular or white) [8].

Another argument against endometriosis as a surgical disease is the importance of the uterus in endometriosis. The uterine endometrium is known to be abnormal in endometriosis, and retrograde menstruation of uterine endometrial glands/stroma through the fallopian tubes and into the pelvis is the major etiologic mechanism for the development of endometriosis [9-11]. Therefore, one wonders how conservative surgery alone could have any long-lasting efficacy, as it involves ablation or excision of endometriosis lesions but does not treat the uterus. However, conservative surgery can be accompanied by other procedures such as pre-sacral neurectomy (division of the nervous supply to the uterus) or intra-operative insertion of the levonorgestrel-releasing intrauterine device (IUD), which have been found to improve pelvic pain when added to conservative surgery for endometriosis $[12,13]$. Alternatively, definitive surgery involving removal of the uterus (hysterectomy), with or without bilateral salpingo-oophorectomy, can be offered as it has low recurrence rates with a $10-15 \%$ risk of re-operation at 5 years [11]. Definitive surgery can be performed laparoscopically in most cases, and remains a choice for women once childbearing has been completed.

Studies on the effectiveness of conservative surgery for endometriosis-related pain are controversial. There is a significant rate of symptom recurrence after conservative surgery for endometriosis, with one study reporting a 50\% risk of re-operation at 5 years [11]. In a Canadian RCT $(n=29)$ with 1 year outcome data on 15 patients, where conservative surgery for endometriosis was compared to placebo laparoscopy (with diagnostic biopsy only), pain reduction was $45 \%$ in the surgery group and $33 \%$ in the placebo group which was not significantly different [14]. However, in the Cochrane review, metaanalysis of another three RCTs comparing conservative surgery to placebo laparoscopy clearly demonstrated that surgical treatment did significantly improve pain at 6 months [15]. This cannot be overlooked, as these were blinded placebo-controlled trials, and such trials for other pelvic pain procedures are either not available (e.g. for uterine suspension) or have shown no impact (e.g. for laparoscopic uterosacral nerve ablation (LUNA) [16]). Notably, the Canadian trial was not combined with the other three RCTs in the meta-analysis [15], seemingly due to differences in how pain outcomes were measured. Based on this Cochrane analysis, the weight of randomized trial evidence appears to support a benefit of conservative surgery for endometriosis pain.

There is also evidence that conservative surgery for endometriosis can increase pregnancy rates in women with infertility; however, assisted reproductive technology (ART) such as in vitro fertilization (IVF) also improves pregnancy rates in endometriosis-associated infertility [17] and some prefer empiric ART to surgical treatment of endometriosis. As well, surgical excision of ovarian endometriosis cysts may reduce ovarian reserve and negatively affect ovarian stimulation, although the impact on ART pregnancy rates is less clear $[17,18]$. On the other hand, a Cochrane review of two RCTs for conservative surgical treatment of minimal-mild endometriosis for infertility did show a fertility benefit for surgery [19] (although on their own, only one study showed a difference [20]). The results of the meta-analysis are modest $(\mathrm{NNT}=12)$, but again cannot be overlooked as surgical treatment was compared to placebo laparoscopy. In addition, larger endometriosis cysts are unlikely to resolve on their own and can present technical challenges for IVF [21], with the most recent committee opinion for the American Society for Reproductive Medicine stating that surgery should be considered for endometriosis cysts $>4 \mathrm{~cm}$ in women planning IVF [22]. Furthermore, surgical excision of endometriosis cysts is known to increase the odds of spontaneous pregnancy in randomized trials [23]. Therefore, surgery for endometriosis and endometriosis ovarian cysts remains a viable option for selected women who prefer a surgical approach to infertility.

Perhaps some of the controversy is that the outcomes of interest, pain and infertility, are outcomes where it can be difficult to achieve an absolute "success". Patients with endometriosis can have a multifactorial

*Corresponding author: Paul Yong, BC Women's Centre for Pelvic Pain and Endometriosis, BC Women's Hospital and Health Centre, 4500 Oak Street Vancouver, BC, Canada V6H 3N1, Tel: 604-875-2445; Fax: 604-875-2569; E-mail: pyong@cw.bc.ca

Received December 23, 2013; Accepted January 28, 2014; Published February 05, 2014

Citation: Yong PJ, William C, Allaire C (2014) Is Endometriosis a Surgical Disease? Surgery Curr Res 4: 164. doi:10.4172/2161-1076.1000164

Copyright: @ 2014 Yong PJ, et al. This is an open-access article distributed under the terms of the Creative Commons Attribution License, which permits unrestricted use, distribution, and reproduction in any medium, provided the original author and source are credited. 
origin of their pain (e.g. painful bladder, irritable bowel, musculoskeletal dysfunction, neuropathic pain, or psychological comorbidity) [24,25], and such patients are unlikely to be completely pain-free with conservative surgery alone. Infertility is also multifactorial, and no treatment for infertility will ever be $100 \%$ successful in achieving pregnancy, with even IVF having limits for per-cycle pregnancy rates [26]. Thus, perhaps expectations for endometriosis surgery need to be more realistic.

Moreover, there is debate on the best method for conservative surgery, with RCTs showing no significant differences between excision and ablation of endometriosis (possibly due to insufficient power) $[27,28]$. But perhaps the focus should be less on the method of conservative surgery, and more on the degree to which the endometriosis was completely treated. For example, one observational study found that complete conservative surgical treatment of endometriosis resulted in higher pregnancy rates, compared to incomplete surgical treatment that left visible residual disease [29]. Thus, the key factor may be the surgeon completely treating all visible endometriosis (regardless of method). Hence, one would expect that ablation and excision of minimal-mild superficial endometriosis would be equivalent, since both methods can completely treat such lesions. In contrast, excision is likely to be superior for the complete treatment of moderate-to-severe deeply infiltrating endometriosis. More studies are needed to confirm whether the concept of complete treatment ("optimal debulking") applies to endometriosis as it does to gynecologic cancers.

In conclusion, we propose that endometriosis remains a surgical disease but with many subtleties and controversies. More research is needed to refine the role of surgery in endometriosis. In the future, endometriosis may be diagnosed by non-invasive means, and novel disease-modifying drugs may become available, but until then, surgery remains indispensible to the diagnosis and management of endometriosis.

\section{References}

1. Leyland N, Casper R, Laberge P, Singh SS; SOGC (2010) Endometriosis: diagnosis and management. J Obstet Gynaecol Can 32: S1-32.

2. Simoens S, Hummelshoj L, D'Hooghe T (2007) Endometriosis: cost estimates and methodological perspective. Hum Reprod Update 13: 395-404.

3. Levy AR, Osenenko KM, Lozano-Ortega G, Sambrook R, Jeddi M, et al. (2011) Economic burden of surgically confirmed endometriosis in Canada. J Obstet Gynaecol Can 33: 830-837.

4. Allaire C (2006) Endometriosis and infertility: a review. J Reprod Med 51: 164-168.

5. Munksgaard PS, Blaakaer J (2011) The association between endometriosis and gynecological cancers and breast cancer: a review of epidemiological data. Gynecol Oncol 123: 157-163.

6. Munksgaard PS, Blaakaer J (2012) The association between endometriosis and ovarian cancer: a review of histological, genetic and molecular alterations. Gynecol Oncol 124: 164-169.

7. Rahmioglu N, Missmer SA, Montgomery GW, Zondervan KT (2012) Insights into Assessing the Genetics of Endometriosis. Curr Obstet Gynecol Rep 1: 124137.

8. Whitehill K, Yong PJ, Williams C (2012) Clinical predictors of endometriosis in the infertility population: is there a better way to determine who needs a laparoscopy? J Obstet Gynaecol Can 34: 552-557.

9. Burney RO, Giudice LC (2012) Pathogenesis and pathophysiology of endometriosis. Fertil Steril 98: 511-519.

10. Carvalho L, Podgaec S, Bellodi-Privato M, Falcone T, Abrão MS (2011) Role of eutopic endometrium in pelvic endometriosis. J Minim Invasive Gynecol 18: 419-427

11. Shakiba K, Bena JF, McGill KM, Minger J, Falcone T (2008) Surgical treatment of endometriosis: a 7-year follow-up on the requirement for further surgery. Obstet Gynecol 111: 1285-1292.
2. Proctor ML, Latthe PM, Farquhar CM, Khan KS, Johnson NP (2005) Surgical interruption of pelvic nerve pathways for primary and secondary dysmenorrhoea. Cochrane Database Syst Rev 4: CD001896.

13. Abou-Setta AM, Al-Inany HG, Farquhar C (2006) Levonorgestrel-releasing intrauterine device (LNG-IUD) for symptomatic endometriosis following surgery. Cochrane Database of Systematic Reviews 4: CD005072.

14. Jarrell J, Mohindra R, Ross S, Taenzer P, Brant R (2005) Laparoscopy and reported pain among patients with endometriosis. J Obstet Gynaecol Can 27 477-485.

15. Jacobson TZ, Duffy JMN, Barlow D, Koninckx PR, Garry R (2009) Laparoscopic surgery for pelvic pain associated with endometriosis. Cochrane Database of Systematic Reviews 4: CD001300.

16. Daniels JP, Middleton L, Xiong T, Champaneria R, Johnson NP, et al. (2010) Individual patient data meta-analysis of randomized evidence to assess the effectiveness of laparoscopic uterosacral nerve ablation in chronic pelvic pain Hum Reprod Update 16: 568-576

17. Falcone T, Lebovic DI (2011) Clinical management of endometriosis. Obstet Gynecol 118: 691-705

18. Somigliana E, Berlanda N, Benaglia L, Viganò $P$, Vercellini $P$, et al. (2012) Surgical excision of endometriomas and ovarian reserve: a systematic review on serum antimüllerian hormone level modifications. Fertil Steril 98: 1531-1538.

19. Jacobson TZ, Duffy JMN, Barlow D, Farquhar C, Koninckx PR, et al. (2010) Laparoscopic surgery for subfertility associated with endometriosis. Cochrane Database of Systematic Reviews 1: CD001398.

20. Marcoux S, Maheux R, Bérubé S (1997) Laparoscopic surgery in infertile women with minimal or mild endometriosis. Canadian Collaborative Group on Endometriosis. N Engl J Med 337: 217-222.

21. Jadoul P, Kitajima M, Donnez O, Squifflet J, Donnez J (2012) Surgical treatment of ovarian endometriomas: state of the art? Fertil Steril 98: 556-563.

22. Practice Committee of the American Society for Reproductive Medicine (2012) Endometriosis and infertility: a committee opinion. Fertil Steril 98: 591-598.

23. Hart RJ, Hickey M, Maouris P, Buckett W (2008) Excisional surgery versus ablative surgery for ovarian endometriomata. Cochrane Database of Systematic Reviews 2: CD004992.

24. Jarrell JF, Vilos GA, Allaire C, Burgess S, Fortin C, et al. (2005) Consensus guidelines for the management of chronic pelvic pain. J Obstet Gynaecol Can 27: 781-826.

25. Jarrell JF, Vilos GA, Allaire C, Burgess S, Fortin C, et al. (2005) Consensus guidelines for the management of chronic pelvic pain. J Obstet Gynaecol Can 27: 869-910.

26. Sunderam S, Kissin DM, Flowers L, Anderson JE, Folger SG, et al. (2012) Assisted reproductive technology surveillance--United States, 2009. MMWR Surveill Summ 61: 1-23.

27. Wright J, Lotfallah $\mathrm{H}$, Jones $\mathrm{K}$, Lovell D (2005) A randomized trial of excision versus ablation for mild endometriosis. Fertil Steril 83: 1830-1836.

28. Healey M, Ang WC, Cheng C (2010) Surgical treatment of endometriosis: a prospective randomized double-blinded trial comparing excision and ablation. Fertil Steril 94: 2536-2540.

29. Suginami $H$, Tokushige $M$, Taniguchi F, Kitaoka $Y$ (2002) Complete removal of endometriosis improves fecundity. Gynecol Obstet Invest 53 Suppl 1: 12-18. 